\title{
MedienPädagogik
}

Zeitschrift für Theorie und Praxis der Medienbildung

Themenheft Nr. 42: Optimierung in der Medienpädagogik.

Forschungsperspektiven im Anschluss an den 27. Kongress der DGfE

Herausgegeben von Patrick Bettinger, Klaus Rummler und Karsten D. Wolf

\section{Hegemonialität und Gouvernementalität von Optimierungsverhältnissen}

\section{Prekäre postdigitale Subjektivierungspraktiken zwischen Affirmation und Widerständigkeit}

Maximilian Waldmann und Ulaş Aktaş

\begin{abstract}
Zusammenfassung
In ästhetisch-medialen Praktiken zeigen sich verschiedene Formen von Optimierungszusammenhängen, die zumeist mit Prozessen der Individualisierung und Vereinzelung gekoppelt sind. Ästhetisch-mediale Praktiken können aber auch Möglichkeiten zur Kollektivierung und zum Widerstand gegen die allgegenwärtigen Optimierungsansprüche bieten. Vor diesem Hintergrund werden sowohl repressive als auch widerständige Seiten ästhetisch-medialer Praxen ins Auge gefasst. Die Ambivalenzen werden an zwei Beispielen beleuchtet. Das erste Beispiel stammt aus Japan und zählt zu den Intelligent Assistent Systemen. Die nach einem Animecharakter gestaltete dreidimensionale Figur, Azuma Hikari, soll das Leben von jungen Single-Männern angenehmer gestalten und übernimmt für sie eine Reihe von Sorgetätigkeiten. An diesem Beispiel wird der technologiegestützte Umgang mit Vereinzelungstendenzen neoliberaler Gesellschaften aus einer care-feministischen Perspektive analysiert. Das zweite Beispiel ist das geplante digitale Mahnmal in der Keupstrasse, das mit Hilfe von augmented reality-Technologie an die beiden Bombenanschläge des NSU in Köln erinnern will. Gegenüber der offensichtlichen Dominanz der servilen Seite der prekären Selbstregierung im ersten Beispiel werden im zweiten Möglichkeiten des Widerstands gegen neoliberale Optimierung und Formen der Stiftung von Gemeinschaft untersucht.
\end{abstract}

Hegemoniality and governmentality of (self)-optimization nexuses. Precarious subjectivation practices in the post-digital age between affirmation and resistance

\begin{abstract}
Aesthetic digital practices reflect various forms of (self)-optimization nexuses, most of which are linked to individualization and isolation processes. However, these practices can also offer opportunities for collectivization and act as a source of resistance to the ubiquitous imperative to self-optimize.
\end{abstract}


Within this context, this article focuses on both the repressive and resistant aspects of aesthetic media practices. Two examples have been selected to illustrate these ambivalences: The first is a Japanese development in the field of intelligent assistant systems. Inspired by an anime character, the three-dimensional Azuma Hikari is designed to brighten the lives of single young men and takes on a range of caring tasks for them. This example is used as the basis for a feminist analysis of gendered care work and how neoliberal societies use technology to address the tendency towards isolation. The second example is the planned digital memorial on Keupstrasse, which will use augmented reality technology to commemorate the two NSU bomb attacks in Cologne. In contrast to the servile face of precarious self- governance, which is clearly the predominating aspect of the first example, the second explores forms of community building and opportunities for resistance to neoliberal optimization.

\section{Einleitung}

Digitalisierung wird gern als die technologische Antwort auf viele Herausforderungen einer durch Heterogenität geprägten Gesellschaft gesehen. Dies täuscht jedoch darüber hinweg, dass sich über Formen der Digitalisierung Machttechniken konstituieren, die nicht auf der Unterdrückung des Individuums, sondern im Gegenteil auf seiner Befreiung beruhen. Gerade die Selbstverwirklichung des Individuums und sein individuelles Lernen bieten die Möglichkeit zur «Führung der Selbstführungen» (Foucault). Dies geschieht nicht gegen seinen Willen, sondern in seinem Eigeninteresse. Diese Form der Macht ist effizienter und produktiver als repressive Formen der Führung. Ihr Zwangsmechanismus liegt in der Verschleierung der Abhängigkeit der Einzelnen von anderen und der Depolitisierung gesellschaftlicher Ungleichheitsverhältnisse. Heterogenität ist allerdings nicht ohne auf Macht und Herrschaft beruhende Ungleichheitsverhältnisse zu verstehen. Das Kalkül der Führung der Selbstführungen ist, dass prekäre Verhältnisse zu mehr Wettbewerb und damit zu grösserer Produktivität führen. In einer Zeit umfassender ökonomischer und sozialer Prekarisierung stehen die Individuen in einem permanenten Wettbewerb miteinander, weil sie durch die kontinuierliche Erfassung von Leistungen und Vergleichsmessungen gegeneinander angeordnet (Sternfeld 2020) und dabei Zugänge zu einem «guten», d. h. einem individuell abgesichertem Leben limitiert werden (vgl. Butler 2012). Der unsichtbare Zwang hinter der Individualisierung besteht gerade in dieser unausgesprochenen Bedrohung durch sozialen Abstieg, die mit Optimierungspraktiken, wie Evaluationen, Rankings und Monitoringverfahren verknüpft ist (vgl. Lorey 2012). Die Vision einer technologisch gestützten Individualisierung mit ihrem Imperativ der Eigenverantwortung, ihren Befreiungsillusionen und dem permanenten Zwang zur Selbstoptimierung kann daher als ein (un-)beabsichtigtes Manöver verstanden werden, um von der Zunahme der Prekarisierung abzulenken. Die Bewältigung gesellschaftlicher Probleme durch 
Selbsttechnologisierung suggeriert dem prekarisierten Selbst, es sei allein verantwortlich für seine Situation. Die Führung der Selbstführungen korrespondiert jedoch mit der Prekarisierung von Lebensverhältnissen. Damit untergräbt die «Regierung durch Prekarisierung» (Lorey 2012) Formen liberaler Kritik und verkennt gesellschaftlich wirksame Machtordnungen. Sie etikettiert Formen der Benachteiligung durch vergeschlechtlichte, klassenbasierte und rassifizierte Privilegstrukturen als individuell gedeutete (Verschiedenheiten) bzw. als (Diversität) und negiert die politische Dimension von Ungleichheit. Diese Herausforderungen lassen sich nicht von einer lediglich affirmativen Sichtweise auf den Zusammenhang von Digitalisierung und Optimierung adressieren. Wir wollen deshalb im Folgenden unterschiedliche Praxisrelationen fokussieren, in denen Zusammenhänge zwischen Optimierung und Digitalisierung in Hinblick auf die jeweils voraus liegenden Macht- und Herrschaftsordnungen deutlich werden. Zwei Fragen leiten dabei unser Erkenntnisinteresse:

1. Welche Bedeutung haben postdigitale Apparaturen bei der Reproduktion von Macht- und Herrschaftsordnungen?

2. Auf welchen Prinzipien sollte eine praxeologische Analyse beruhen, die sich postdigitalen Optimierungsverhältnissen aus einer ungleichheitssensiblen Perspektive annähert und Spielräume von gegenhegemonialen Praktiken beschreiben kann?

Zur Beantwortung dieser Fragen werden wir in einem ersten Schritt zwei dominante Kontexte von Optimierung skizzieren, die wir als Regime bezeichnen (1). Regime regulieren nicht nur Machtverhältnisse zwischen Subjekten und Gesellschaft. In Regimen werden darüber hinaus hegemoniale Relationen der Über- und Unterordnung organisiert. In einem zweiten Schritt werden wir anhand von zwei Beispielen für postdigitale Praxisverhältnisse die Beziehungen zwischen Prozessen der Individualisierung und Optimierungszwängen untersuchen (2). Dabei werden wir sowohl auf Faktoren eingehen, die Machtverhältnisse in den Praktiken sedimentieren, als auch Subjekt-Technologie-Interaktionen analysieren, die sich diesen Zwängen entziehen. Im letzten Arbeitsschritt erfolgt eine Bilanzierung und Gegenüberstellung der analysierten Beispiele im Hinblick auf ausgewählte Kriterien, die wir für eine ungleichheitssensible Praxeologie als relevant erachten (3).

\section{Regime und postdigitale Praktiken}

Nachfolgend wollen wir anhand von zwei Analysebeispielen Subjektivationsverhältnisse in unterschiedlichen Regimen unter den Bedingungen von Optimierung in den Blick nehmen. Regime stellen für ein Netz von bedeutungserzeugenden Elementen Verknüpfungsregeln bereit (vgl. Dimitrova et al. 2012, 9-12). Regime regulieren dadurch das Verhältnis von Subjekten zu sich selbst, zu anderen und zum Dritten der 
Ordnung. Sie figurieren Relationen der Unter- und Überordnung, beeinflussen, wer oder was auf welche Weise sicht- und sagbar wird und flexibilisieren oder verengen Spielräume der Subjektivierung. Wird Optimierung im Zusammenhang mit Regimen betrachtet, dann verläuft sie also stets im Wirkungsfeld von kulturellen wie politischökonomischen Dominanzverhältnissen. Durch den Bezug auf diese Verhältnisse lässt sich kennzeichnen, dass Wirkungsweisen und Bedeutungen der Optimierung jeweils regimespezifisch ausgeprägt sind und entsprechend variieren (näheres dazu in den Abschnitten 1.1 und 1.2). Zu den Elementen, die in Regimen miteinander verknüpft werden, zählen symbolische, materielle und, so werden wir im Folgenden durch die Analyse der Beispiele erläutern, postdigitale Praktiken. Unter 〈Postdigitalität` verstehen wir im Anschluss an Jeremy Knox (2019) zeitliche, strukturelle und analytische Bedingungen der Möglichkeit, Subjekt-Technologie-Arrangements und die Transformation von Relationen zwischen Digitalität und Gesellschaft im Anschluss an die Normalisierung und weitere Beschleunigung der Digitalisierung zu begreifen. Nicht nur befinden wir uns an einem Punkt, an dem der Umgang mit digitaler Technologie ‘normalı geworden ist. Darüber hinaus sind digitale Medien und Apparaturen mit ihren Umwelten in einer Weise verzahnt, dass die Unterscheidung in eine ‘digitale) und eine «nicht-digitale) Sphäre kaum sinnvoll zu treffen ist. Postdigitalität bezeichnet mithin eine Kritik gegenüber dominanten Diskursen der (digitalen Transformation), darunter auch die unhinterfragten Relationen zu neoliberalen Optimierungszwängen (vgl. auch Dander 2020). Postdigitale Praktiken sind demnach solche, die die Unterscheidung in eine digitale und eine nicht-digitale Sphäre unterlaufen. Darüber hinaus sind postdigitale Praktiken auch daran zu erkennen, dass sie eine kritische Distanzierung gegenüber gesellschaftlichen Machtkalkülen und Herrschaftsmustern vollziehen, indem sie beispielsweise ein Gegenmodell zu unseren bisherigen Ordnungen des Sicht- und Sagbaren entwerfen und damit zu anderen Subjektivierungspraktiken führen können.

\subsection{Repräsentationsregime}

Unter Repräsentationsregimen verstehen wir im Anschluss an Stuart Hall (1994) eine spezifische Anordnung von bildlichen und anderen repräsentierenden Elementen und Praktiken, die Verhältnisse zwischen Unter- und Überordnung, zwischen Täterund Opferschaft und zwischen (den Anderen) und (uns) erzeugen. Dabei handelt es sich nicht einfach um eine Abbildungsfunktion visueller Arrangements, sondern es werden hegemoniestabilisierende Differenzen durch ethnisierende, kulturalisierende, vergeschlechtlichende u. a. Unterscheidungspraktiken gebildet. So liesse sich etwa die mediale Darstellung der Vorfälle in der Silvesternacht 2015/16 vor dem Kölner Dom als Beispiel für ein Repräsentationsregime nennen. Die Rassialisierung von Täter-Opfer-Verhältnissen durch die Berichterstattung konzentrierte sich schnell auf 
die Herkunft der Täter und löste eine Debatte über schärfere Abschieberegelungen aus (vgl. Hark und Villa 2017). Sexualisierte Gewalt erschien dadurch vorwiegend als Problem islamisch geprägter Gesellschaften, was strukturell zu einer Reaktivierung des neokolonialen Motivs vom <unzivilisierten Orient> und einer Aufwertung europäisierter Kulturen führte (vgl. bereits Said 1978). Der Begriff des Repräsentationsregimes erlaubt es hier, anti-muslimischen Rassismus im Wirkungsfeld der Machtkontinuitäten des Kolonialismus und der Orientalisierung der (Anderen` in den alltäglichen Repräsentationspraktiken zu analysieren. Allen Repräsentationsregimen ist nämlich gemeinsam, dass sie unser Verhältnis zu den ‘Anderen` durch Othering-Praktiken hervorbringen. Optimierung bezieht sich in Repräsentationsregimen auf den Zusammenhang zwischen Symbolen, z. B. in medialen Darstellungsformaten wie der Berichterstattung, und hegemonialen Bedeutungsstrukturen. Je näher bedeutungserzeugende Prozesse der Enkodierung und Dekodierung von Symbolen dominanzgesellschaftlichen Prinzipien stehen, wie etwa die Vorverurteilung von muslimischen Anderen u.a. rassistische Praktiken, desto optimaler lassen sie sich durch Massenmedien verbreiten (vgl. Hall 1994). Jeder Akt der Dekodierung, der von den übermittelten Codes abweicht - also «nur einen geringen oder keinen Deckungsgrad mit diesen aufweist» - kann «als oppositionell bezeichnet werden» (Marchart 2008, 146). Die Rezipierenden (Empfänger) sind im hegemonietheoretischen Kommunikationsmodell in die Verleihung und Modifikation von Bedeutungen aktiv involviert. Die Bedeutung von Symbolen wird in Aushandlungsprozessen zwischen verschiedenen (gegen-) hegemonialen Codes als Äquivalenz (Reproduktion von Hegemonie) oder als Differenz (oppositionelle Position zu Hegemonie) zwischen Enkoder und Dekoder fortgeschrieben oder verändert. Es geht hierbei nicht darum, Subjekte oder sich selbst einfach nur an herrschende Normen der Selbstführung anzupassen, sondern um eine bessere Passung von bedeutungserzeugenden Praktiken mit dominanzgesellschaftlichen Prinzipien. Halls medienbezogenes Hegemoniemodell berücksichtigt allerdings nicht die Spezifität von digitalen Technologien. In digitalen Repräsentationsökonomien lassen sich die Praktiken der Produktion/Distribution/Rezeption bzw. das Enkodieren und Dekodieren nicht mehr trennscharf voneinander unterscheiden, wie dies bei den Massenmedien TV, Radio, Zeitung noch der Fall war (vgl. Terranova 2000), was auch daran liegt, dass User digitaler Medien stärker in alle der genannten Momente aktiv involviert sind. Die Unterminierung der von Hall vorgeschlagenen Unterscheidung impliziert den Übergang vom klassischen Enkoder-Dekoder-Modell zu einem postdigitalen diskursiv-praxeologischen Modell der Bedeutungserzeugung (siehe 2.). Die Relationalität der (hegemoniekonformen oder opponierenden) postdigitalen Vollzüge, so werden wir in unseren Analysen kennzeichnen, geht den durch sie konstituierten Relata (Subjekte, digitale Artefakte) stets voraus. 


\subsection{Regime der Prekarisierung}

Mit Hilfe des Regimes der Prekarisierung lassen sich die bisher herausgestellten Überlegungen zu hegemonialen Praktiken für neoliberale Gesellschaften gouvernementalitätstheoretisch aktualisieren. Nach Isabel Lorey (2012), die Formen der Prekarität durch Unterordnung, Exklusion und Bedrohung der leiblichen Existenz (z. B. durch rassistische Gewalt) von Formen der flexiblen Selbstregulierung und Arbeitsteilung als Anpassung an eine liberal-bürgerliche Subjektnorm unterscheidet, bezeichnet Prekarisierung eine Form des Regierens und Regiert-Werdens, die auf die Allgegenwart von Unsicherheit in globalisierten Gesellschaften verweist. Prekarisierung als Regierungsinstrument zu begreifen hiesse, die Normalisierung von Prekarität im Kontext eines neoliberalen Sicherheitsdiskurses des Staates zu sehen, der sich aufgrund von umfangreichen Ökonomisierungsprozessen aus vielen sozialen Teilbereichen zurückzieht, aber gleichzeitig polizeilich-militärische Disziplinierungs- und Kontrollformen zur Innen- und Aussensicherung fördert (ebd., 85). Heutige liberalstaatliche Diskurse beruhen auf einem androzentrisch-bürgerlichen Sicherungsideal in der Nachfolge von Thomes Hobbes. Dies hat zur Folge, dass staatliche Institutionen in flexible Marktlogiken nur noch dann intervenieren, wenn die Gesamtnormalität gefährdet ist - etwa durch die drohende Gefahr eines Bürgerkrieges, der durch zu grosse Verteilungsungerechtigkeiten wahrscheinlicher werden könnte, oder den Ausbruch einer Pandemie. Pointiert liesse sich das Credo des Prekarisierungsregimes wie folgt auf den Punkt bringen:

«Freiheit wird nicht prinzipiell staatlich beschränkt, Unsicherheit nicht prinzipiell staatlich bekämpft, sondern beide werden zur ideologischen Voraussetzung für gouvernementale Prekarisierung.» (ebd., 86)

Und das bedeutet speziell für Gesellschaften des globalen Nordens, dass der freiwillige Zwang zur Optimierung durch kreative Selbstbewirtschaftung und die Effizienzsteigerung von Feedbackschleifen nicht im Widerspruch zu rassistisch unterlegten Ausgrenzungsformen und neuen, vergeschlechtlichten und klassenbasierten Ungleichverteilungen von Arbeitslasten stehen. Es zeigt sich vielmehr, dass das neoliberale Duo aus flexibler Freiheit und (Un-)Sicherheit das Prekärwerden von Subjektverhältnissen nachgerade evoziert. Der Grund dafür ist darin zu sehen, dass Ungleichheiten in Gestalt einer Verflechtung beider Prekaritätsformen integraler Bestandteil der Marktdynamiken sind und von diesen sogar verstärkt werden. Optimierung bezieht sich im Rahmen des Prekarisierungsregimes von daher besonders auf Praktiken des Schutzes vor Unsicherheit und Prekarisierung. Je grösser die Bedrohung durch Prekarität wird, desto mehr wird das Subjekt gezwungen, sich selbst in marktkonformer wie depolitisierender Weise zu optimieren. Der gouvernementale Aspekt der Prekarisierung besteht darin, dass sich ihre Effekte nicht nur in repressiven und rasternden Funktionen zeigen, sondern genauso in ihren ambivalent-produktiven Momenten. 
Prekarisierung stellt insofern sowohl die Bedingung als auch den Effekt von Herrschaft dar, wie Optimierung auf Formen der Herrschaft beruht, die sie selbst erzeugt. Durch den Blick auf das Prekarisierungsregime wird ersichtlich, wie Herstellungsweisen von Schutz und (postdigitale) Technologien der Selbstführung miteinander verknüpft sind. Dadurch, so wollen wir zeigen, lassen sich die Ambivalenzen von Unterwerfung und individueller Ermächtigung problematisieren und der Zusammenhang von Optimierung und konformer Selbstgestaltung kontextspezifisch untersuchen.

\section{Analysebeispiele}

Ein Schauplatz der neoliberalen Verschränkung von Freiheitsgewinnen einiger mit der Ausbeutung und Unfreiheit anderer zeigt sich in unserem ersten Beispiel, in dem es um ein digitales Assistenzsystem aus Japan für junge alleinstehende Männer geht. Das System involviert seine User auf spielerische Weise in neoliberale Geschlechterlogiken, in denen ein Teil der prekären Bedingungen des Subjektseins ausgelagert und an weibliche Subjekte als Sorge-Arbeit delegiert wird. Die User des Systems, das für sie ausgewählte emotionale und planerische Sorgearbeiten verrichtet, profitieren davon, weil ihnen dadurch mehr Kapazitäten für die Bewältigung ihres eigenen prekären Arbeitsalltags zur Verfügung stehen. Anhand dieser Form der Mensch-Maschine-Arbeitsteilung offenbart sich, inwiefern Regime der Prekarisierung weder das von ihnen hervorgerufene Problem der Vereinzelung lösen, noch die Bedingungen von Optimierung selbst erzeugen können. Gleichsam werden eben jene prekären Bedingungen, zu denen Vulnerabilität und Sorgeabhängigkeit zählen, von den flexiblen Subjektivierungsweisen der Optimierung immer wieder stillschweigend vorausgesetzt. Die Analyse wird darauf hinauslaufen zu zeigen, wie die Optimierungszusammenhänge durch digitale Technologien gestützt werden und dadurch keine offensichtlichen Potenziale für widerständige Subjektivierungspraktiken aufbieten.

Das zweite Analysebeispiel ist das digitale Mahnmal Keupstraße in Köln-Mühlheim, das sowohl in seinem widerständigen Verhältnis zum Repräsentationsregime als auch in Relation zum Regime der Prekarisierung analysiert wird. Indem das Mahnmal die Täter-Opfer-Umkehr nach den Anschlägen sichtbar macht, bildet es einen Gegenraum zum Blick- und Urteilsregime der rassifizierten (polizeilichen) Anrufungsordnung. Die postdigitalen Praktiken des Mahnmals widersetzen sich den neoliberalen Optimierungslogiken, indem sie sich den forcierten Entsolidarisierungsprozessen und der Ökonomisierung, Vereinzelung und Kontrolle sozialer Beziehungen entgegenstellen und so den Raum öffnen für Kollektivierungspraktiken und Formen des Sich-umeinander-Kümmerns. 


\subsection{Analysebeispiel ‘Azuma Hikari>}

Die folgenden explorativen Überlegungen zur Individualisierung und Optimierung werden anhand eines Beispiels aus Japan entwickelt. Es stammt aus dem Bereich der Intelligent Assistent Systeme und soll das Leben von jungen Single-Männern angenehmer gestalten, wie in einem Werbevideo der Entwicklerfirma demonstriert wird.

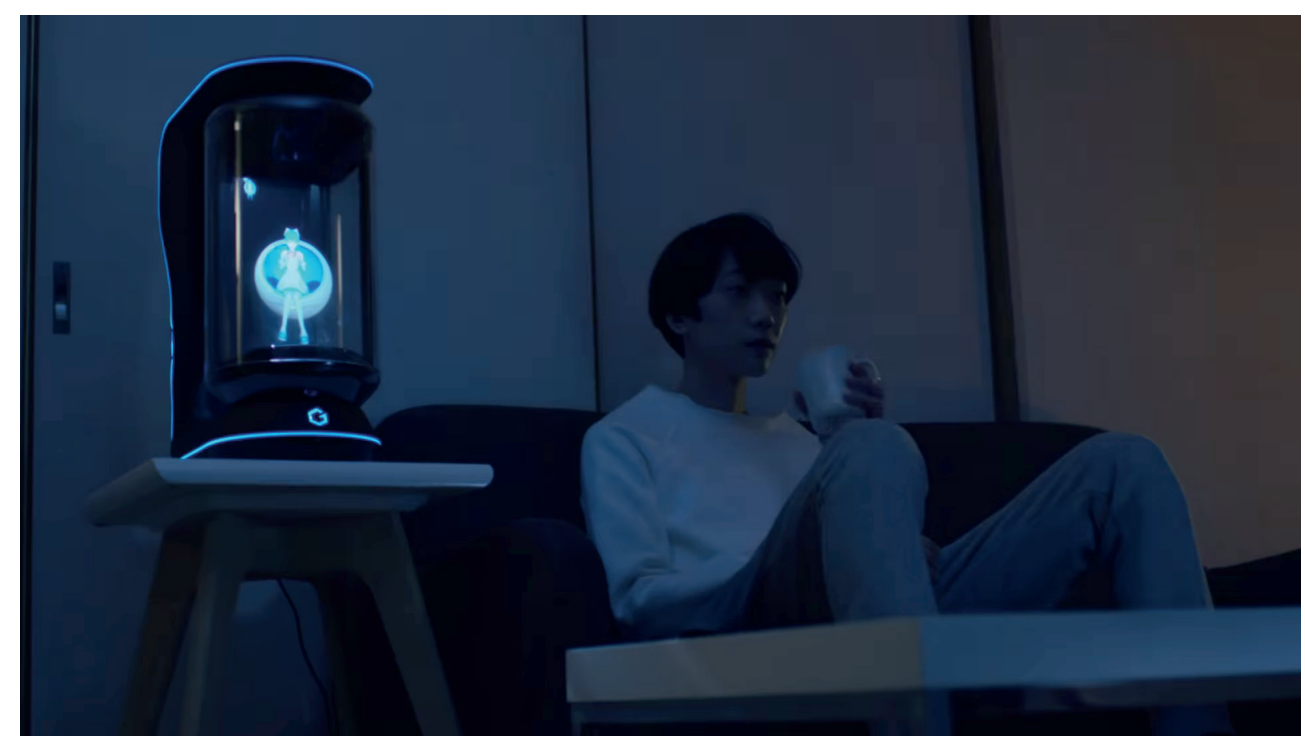

Abb. 1.: Gatebox - Promotion Movie «OKAERI»_english (Screenshot aus dem Video: $1 \mathrm{~m}$ 28s; https://www.youtube.com/watch?v=nkcKaNqfykg).

Das System ist in einem Glaszylinder untergebracht, in dem eine 3D-Projektion der nach einem Anime- oder Spielcharakter gestalteten Figur Azuma Hikari darauf wartet, ihren «Meister) (traditionelle japanische Anredeform) zu wecken. Sie sorgt nicht nur dafür, dass dieser nicht zu spät zur Arbeit kommt. Sie kümmert sich auch nach einem «Blick〉 auf den Wetterbericht darum, dass der Regenschirm nicht vergessen wird und verabschiedet ihren Besitzer, der über eine Taste an dem Glaszylinder oder per Smartphone mit Azuma kommuniziert. Azuma begleitet den User auch über den gesamten Arbeitstag hinweg mit ihren Textnachrichten, indem sie beispielsweise nicht nur viel Spass bei der Arbeit wünscht, sondern sich auch nach dessen Heimkehr erkundigt und diese bisweilen sogar einfordert, um emotionale Nähe zu simulieren.

Anhand dieses Systems, das eine Mischung aus Social Robot und Emotional Intelligent Assistent darstellt, lassen sich exemplarisch eine Reihe von Überlegungen artikulieren, die auf ausgewählte Zusammenhänge zwischen Optimierung, gesellschaftlicher Ordnung und Subjektivierung in Mensch-Maschine-Interaktionen fokussieren. 


\subsubsection{Vergeschlechtlichung als Bedingung von Optimierungsprozessen}

Anhand der Subjekt-Maschine-Interaktionsbeziehung wird deutlich, inwiefern Optimierung stets an bestimmte Voraussetzungen gebunden ist, die oftmals selbst gar nicht Teil von Optimierungsprozessen sind oder von diesen sogar unsichtbar gemacht werden. Im Beispiel sind dies Formen von vergeschlechtlichter Arbeitsteilung: Azuma verrichtet emotionale und planerisch-vorausschauende Sorgeaufgaben, die materiell, symbolisch und sozialnormativ Frauen bzw. der als 'weiblich) codierten Häuslichkeit zugeschrieben werden. Unterstützt wird diese Aufteilung von Privatheit und Öffentlichkeit durch die materielle Beschaffenheit des Systems. Es kann zwar nach draussen kommunizieren, operiert jedoch von zuhause aus und ist schon allein aufgrund der Ausmasse des Glaszylinders nicht sonderlich mobil. Gleichzeitig bleibt die Projektion von Azuma an den Glaskasten gebunden, den sie nicht verlassen kann. Der junge Mann in dem Video soll nicht nur für den Arbeitstag vorbereitet werden, sondern er soll auch ein Gefühl von Geborgenheit bei seiner Rückkehr nach Hause bekommen. Die beschriebene Retraditionalisierung in den interaktiv hergestellten geschlechtlichen Rollenverhältnissen ist durchaus funktional: Das davon profitierende männliche Subjekt der Individualisierung, so lässt sich im Anschluss an Isabell Lorey (2012, 41-43) sagen, ist dann optimal für die Herausforderungen seiner Umwelt gewappnet, wenn jemand oder etwas dafür sorgt, dass die körperlichen Bedingungen seines Subjektseins - Abhängigkeit von Anderen, Angewiesenheit auf (Für-)Sorge, Emotionalität und Vulnerabilität der eigenen Existenz - nicht mehr innerhalb der subjektzentrierten Selbstregierungspraxis thematisch werden müssen. Dazu muss Sorgearbeit ausgelagert und an andere(s) delegiert werden. Care-Work, die sich eben jenen prekären Bedingungen des Subjektseins widmet und damit die Voraussetzung jeder (optimierten) Selbstsorgepraxis darstellt, wird in dem Beispiel auf basale Weise an die (digitale Frau delegiert. Durch diese Form der automatisierten Assistenz wird zum ersten die materielle Dimension der Sorgearbeit unsichtbar gemacht, die nach wie vor, und d. h. in neoliberalen Gleichstellungsgesellschaften, zum Grossteil von Frauen erledigt wird. Zweitens wird hier das Phantasma neoliberaler Selbstregierung einer «möglichst souveräne[n] Beherrschung des «eigenen> Prekärseins» (ebd., 42) weiter verstärkt.

\subsubsection{Kulturspezifische Grenzen von Optimierung?}

Muss jedoch, so liesse sich einwenden, nicht auch der kulturspezifische Kontext bei der Deutung beachtet werden? Bei den Worten «Okaeri nasai», mit denen Azuma den User bei seiner Heimkehr begrüsst, handelt es sich um eine Willkommensformel, die zu einem traditionellen japanischen Ritual gehört. Nach Hause zu kommen wird dabei regelrecht zelebriert. Im Video wird dieses Ritual dadurch begangen, indem Azuma per Netzwerksteuerung Licht und Klimaanlage in der Wohnung einschaltet und für einen ‘warmen〉 Empfang sorgt. Der Einsatzzweck des gendered Social Robot 
beschränkt sich somit nicht allein auf Optimierung, da bei der Deutung die japanspezifische Zielgruppe der jungen alleinstehenden Männer mitberücksichtigt werden muss. Sie finden aufgrund von klassengebundenen Schranken, Zeitmangel durch exzessives Arbeiten und den spezifisch urbanen Sozialisationsstrukturen keine Partnerin, wodurch sie zunehmender Isolation ausgesetzt sind (Ronald und Hirayama 2009). Die kulturalistische Deutung und der mit ihr figurierte westliche Blick auf das «technologiefreundliche Japan» hat jedoch Grenzen, insofern heterosexuelle Skripte und das Gendering von digitalen Systemen überkulturelle und d. h. strukturelle Kennzeichen spätmoderner Geschlechterregime sind, die auch in zahlreichen anderen Apps und vergleichbaren Personal Intelligent Systems global zu finden sind (Ranga und Etzkowitz 2010).

\subsubsection{Welches Geschlecht hat das Subjekt der Optimierung?}

Soziale Ungleichheitsverhältnisse werden in vielen Fällen fast eins zu eins in HighTech-Tools transformiert. Gesellschaftliche Hierarchien schreiben sich so im Digitalen fort, wie Victoria Eubanks (2018) in ihrem kürzlich erschienenen Werk «Automating Inequality» herausarbeitet hat. Im Hinblick auf die Reproduktion von soziosymbolischen Herrschaftsverhältnissen durch digitale Systeme erörtert die Bloggerin und Designwissenschaftlerin Elvia Vasconcelos (2018), weshalb weibliche Charaktere oftmals zur Vorlage für Assistenztechnologien werden. Sie verweist in diesem Zusammenhang auf Laurie Penny, die fragt, wieso wir Robotern weibliche Namen geben. Ihre Antwort darauf lautet: «Weil wir ihre Gefühle nicht beachten wollen». Dabei spielt Penny bewusst mit der Ambivalenz, ob sich der Terminus «ihre Gefühle» auf vergeschlechtlichte KI-Systeme oder Frauen bezieht:

«Right now, as we're anticipating the creation of Als to serve our intimate needs, organise our diaries and care for us, and to do it all for free and without complaint, it's easy to see how many designers might be more comfortable with those entities having the voices and faces of women. If they were designed male, users might be tempted to treat them as equals, to acknowledge them as human in some way, perhaps even offer them an entry-level salary and a cheeky drink after work.» (Penny 2016)

Im Umkehrschluss lässt sich fragen, ob das Geschlecht in sozialnormativer und symbolischer Hinsicht, das von solchen Assistenzsystemen in der Regel profitiert und gleichermassen adressiert wird, stets dem aktiven männlichen Lifestyle- und Erwerbssubjekt entspricht.

\subsubsection{Praxeologie vergeschlechtlichender Subjekt-Maschine-Interaktionen}

Aus Sicht einer medienpädagogischen Sozialisationsforschung, die solche SubjektMaschinen-Ensembles aus einer praxeologischen Perspektive erforscht, sollten wir 
nicht jedoch allein nach «dem> Subjekt der Optimierung fragen. Jutta Weber und Corinna Bath $(2007,62)$ folgend, auf die Vasconcelos gegen Ende ihres Blogs verweist, basiert eine geschlechtersensible Sicht auf Subjekt-Maschine-Interkationen darauf, das pädagogische Verhältnis näher zu untersuchen, das hier figuriert wird. Wer lernt von wem auf welche Weise, wenn wir unsere Maschinen derart cerziehen), dass sie zu Care-Workerinnen und Optimierungs-Assistentinnen werden? Zur Disposition steht dabei allerdings - und hier handelt es sich um eine Forschungslücke im medienpädagogischen Diskurs -, wie sich in praxeologischen Ansätzen die skizzierten gesellschaftlichen Herrschaftsverhältnisse als Teil der Subjekt-MaschineInteraktionspraktiken berücksichtigen lassen. Wenn wir Technologien als ko-konstitutive Elemente in Subjektivierungsvollzügen fassen, wofür Heidrun Allert, Michael Asmussen und Christoph Richter (2018) plädiert haben, so richten wir den Fokus auf die performativen Vollzugswirklichkeiten, die ontologisch jeder vereinzelten Attribuierung auf (das Subjekt) und (die Technik〉 vorausgehen. Mit Patrick Bettinger lässt sich diese Sichtweise, bei der Relationen den Relata stets vorausgehen, um die Dimension der Materialität jeder ko-konstitutiven Praxis ergänzen. Analytisch käme es dann darauf an, im Anschluss an neuere feministische und Perspektiven des New Materialism «den [...] Fokus auf materiell-diskursive Praktiken von Hybridakteuren zu richten, die aus den dynamischen Verflechtungen von menschlichen und nichtmenschlichen Grössen hervorgehen» (Bettinger 2020, 64).

Kombiniert man diese Überlegungen mit dem eingangs erläuterten Regimeverständnis, so lassen sich drei Grundprinzipien für die Erforschung von materiell-diskursiven Optimierungspraktiken festhalten:

1. Die Relation von Subjektwerdung und digitalen Apparaturen zueinander ist von «zweierlei Herkunft (Hybridität).

2. Die miteinander verbundenen Relata gehen nicht aus einem Gleichheitsverhältnis heraus eine Beziehung zueinander ein, da sie stets von gesellschaftlichen Ungleichheitsbeziehungen beeinflusst werden (Asymmetrie).

3. Und weil sich diese Praktiken stets im Kontext einer Ordnung vollziehen (oder diese bisweilen herausfordern), ist Subjektwerdung nicht als zweistellige sondern nur als eine dreistellige Relation zu begreifen (Triadizität).

Hybridität, Asymmetrie und Triadizität, so lautet die Antwort auf unsere zweite forschungsleitende Frage, gehören also zu den Kernprinzipien einer ungleichheitssensiblen Praxeologie.

In Hinblick auf das vorgestellte Beispiel bedeutet dies, Hybridität und Materialität der Subjektivierungsvollzüge in Bezug auf die beiden eingangs skizzierten regimespezifischen Optimierungsweisen zu untersuchen. In dieser Analyseperspektive setzt sich das interaktionale Praxisverhältnis zwischen User und Azuma aus zwei Doppelrelationen zusammen: 
a. Optimierungsrelation 〈Azuma - Repräsentationsregime - User`: Die Lernvollzüge von Azuma folgen einem gendered deep learning, bei dem Algorithmen auf veränderte Umweltbedingungen so reagieren sollen, dass nicht allein die verrichteten Sorgearbeiten, sondern auch die relationale «Weiblichkeit), die dabei emuliert und gleichzeitig auf hegemoniale Weise repräsentiert wird, optimaler zu den Anforderungen und Bedürfnissen des Nutzers passen. Neu ist daran nicht, dass «Sexism, racism and other forms of discrimination are being built into the machine-learning algorithms that underlie the technology behind many (intelligent) systems» (Crawford 2016). Vielmehr besteht der neue, postdigitale Aspekt des Lernens darin, dass digitale Assistenzsysteme ihren Charakter selbst noch besser an die Feedbacks anpassen, die sie von den vorwiegend männlichen Usern in Interaktionen erhalten. Übereinstimmungen mit den praktisch aktualisierten hegemonialen Erwartungen an Weiblichkeit stellen solche Systeme, wie Azuma, dadurch her, indem sie quasi eigenständig ein Selbst-Othering performen.

b. Optimierungsrelation (User - Regime der Prekarisierung - Azuma): Hybridität zeigt sich in dem Beispiel also in Bezug zu den jeweiligen Machtregimen. Denn sowohl das Verhältnis von Azuma zum Repräsentationsregime als auch die Relation zwischen User und dem Regime der Prekarisierung ist von zweierlei Herkunft: Während sich Azuma wie eine vergeschlechtlichte Andere zu verhalten lernt, kann sich der User ganz auf die Anforderungen im Job einstellen, indem er sein Lernselbstverhältnis nach dem für Prekarisierungsregime charakteristischen Modell der kompetenten Selbstführung gestaltet. Durch diese «Selbstcyborgisierung» (Müller 2010, 154) nähert er sich dem Ideal maschinellen Lernens an, das durch ausgewählte Anregungen (‘Nudges〉) und Direktiven von Azuma immer wieder verfeinert werden soll. Dabei geht es nicht darum, was gelernt wird. Ziel ist stattdessen eine effektive Bewirtschaftung des funktionalen Selbstbezuges für alle anstehenden Herausforderungen. Das Verhältnis zum Regime der Prekarisierung zeigt sich nicht nur darin, dass der User im Video Fürsorge erhalten und Häuslichkeit empfinden kann, weil jemand da ist, wenn er nach Hause zurückkehrt. Geschützt vor häuslicher und emotionaler Isolation kann sich der User auch in der Rolle desjenigen erleben, der Azuma finanzielle und emotionale Absicherung bietet, indem er ihr beispielsweise hin und wieder kleine Geschenke macht oder sie sgemeinsam> Abendessen und einen Film schauen. Die Relation zwischen beiden basiert daher auf einer (simulierten) wechselseitigen Abhängigkeit von interaktionalen Selbstversicherungspraktiken gegenüber der Prekarisierung und Unsicherheit der eigenen Existenz. 
Das Fazit der triadischen praxeologischen Perspektive auf die untersuchten postdigitalen Optimierungspraktiken lautet, dass Azuma und ihr User ihre jeweiligen Feedbackschleifen anhand der miteinander verwobenen Relationen zu den Regimen der Repräsentation (a) und des Prekären (b) miteinander synchronisieren.

\subsection{Analysebeispiel Digitales Mahnmal in der Keupstraße}

Im zweiten Beispiel werden ästhetisch-mediale Praktiken fokussiert, die radikal-demokratische Fragen nach Kollektivierung aufwerfen und gegen ein rassistisches Repräsentationsregime opponieren. Das geplante digitale Mahnmal in der Keupstraße, das mit Hilfe von Augmented-Reality-Technologie an die beiden Bombenanschläge des Nationalsozialistischen Untergrunds (NSU) im Jahr 2004 in Köln erinnern soll, ermöglicht sowohl Formen der Solidarisierung und des Widerstands gegen strukturellen Rassismus ins Auge zu fassen, als auch für taktische Spielräume der Widerständigkeit innerhalb neoliberaler Optimierungsordnungen aufmerksam zu werden.

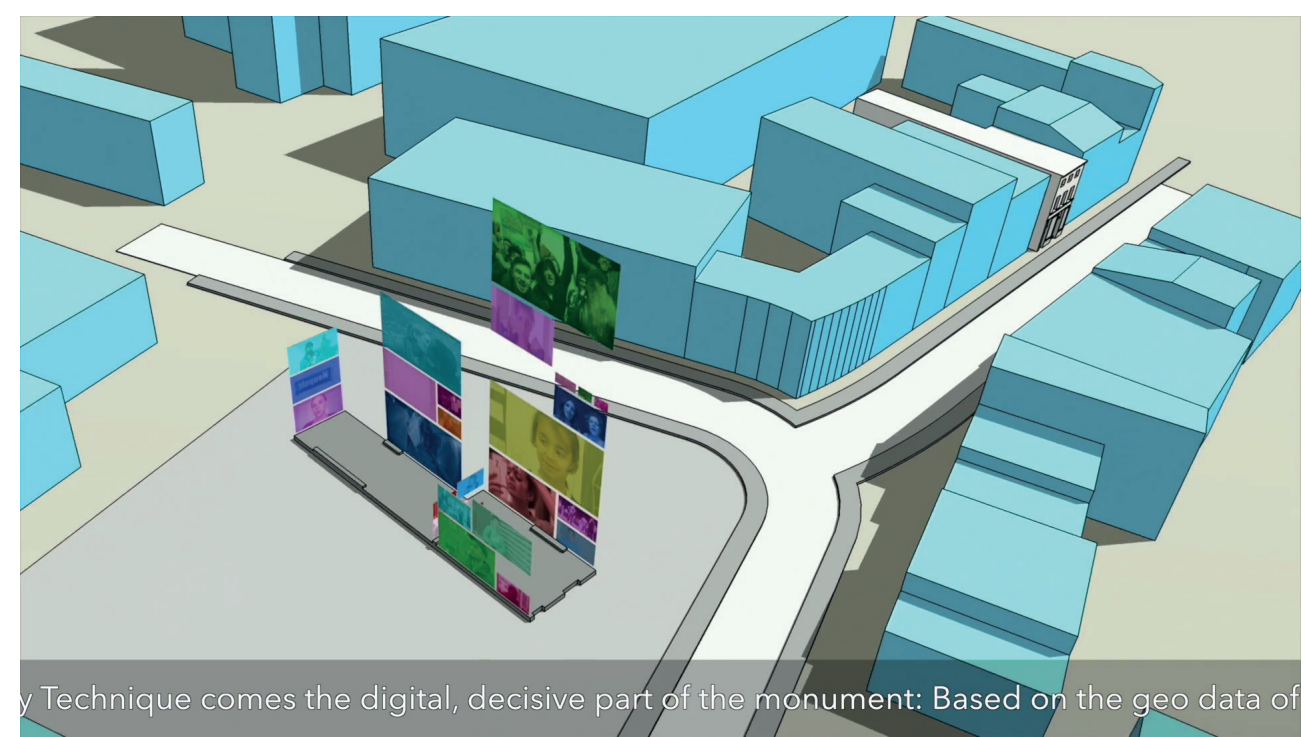

Abb. 2.: Das Mahnmal als virtuelles Haus (Screenshot Video der Initiative 1min 28s; https://player.vimeo.com/video/411758828).

Das digitale Mahnmal wurde von dem Künstler Ulf Aminde und der Initiative «herkesin meydani/Keupstrasse ist überall» entworfen und soll in Köln-Mühlheim entstehen. Es besteht aus einer Betonbodenplatte, die den Grundmassen des Hauses in der Keupstr. 29 entspricht, an dem die Bombe am 9. Juni 2004 explodiert ist. 


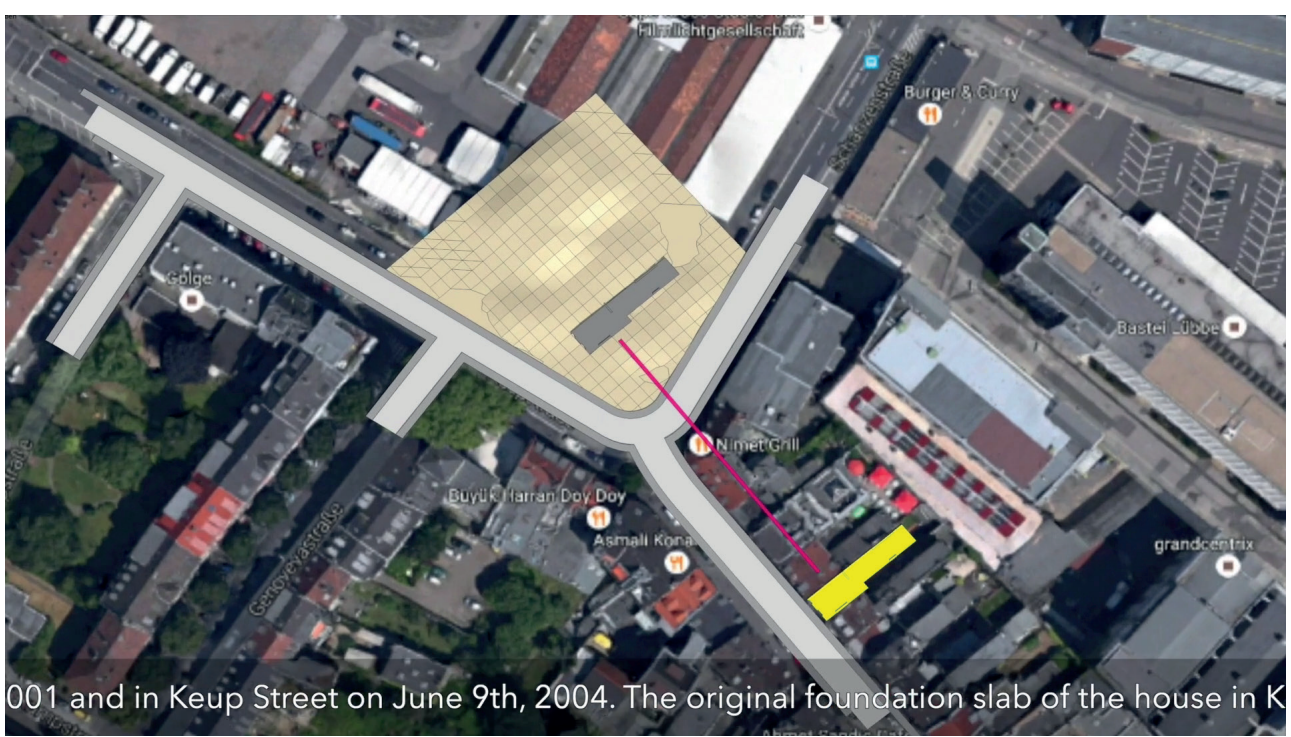

Abb. 3.: Mahnmal in der Sichtachse des Friseursalons (Screenshot aus dem Video: 39 sec; https://player.vimeo.com/video/313851026).

Auf dem Bild ist das Haus Keupstraße 29 gelb und das Mahnmal grau abgebildet. Mittels Augmented-Reality-Technologie lässt das Mahnmal das Haus virtuell wiedererstehen. Die Wände bestehen dabei aus Videos, die aktiviert und z. B. auf einem Smartphone angeschaut werden können. Weitere Videos können hinzugefügt werden, so dass das Haus immer weiterwachsen kann. Dabei geht es nicht um die (immersive) Simulation einer Bildwirklichkeit oder allein um informative Daten zu den beiden Kölner Anschlägen. Vielmehr soll hier die Erfahrung von Migrantinnen und Migranten sichtbar gemacht und als Spur in den politisch-medialen Raum eingeschrieben werden. Im Sinne des mit Knox skizzierten Verständnisses einer immer hegemonial situierten «postdigitalen Kondition> unterläuft das Mahnmal die Unterscheidung von Digitalität und Nichtdigitalität und interveniert in den Raum des Politischen. Das Mahnmal macht das politisch Unsichtbare sichtbar, indem es die augmentierten diskursiven Dimensionen des Ortes Keupstraße wachruft. Auf diese Weise soll ein Raum der Vielen ${ }^{1}$ entstehen, wie die Initiative betont, und der generalisierende Blick auf den Platz ebenso wie das allägliche Durchqueren des Ortes gestört werden. Wie auch schon in der ersten Beispielanalyse werden wir auch hier eine triadische praxeologische Perspektive verfolgen.

Wir wollen also auch hier ergründen, inwiefern Subjektwerdung und materielldiskursive Praktiken über spezifische Herrschaftsverhältnisse in Beziehung zum Dritten der Ordnung stehen. Wie schon im Beispiel zuvor verstehen wir Ordnung als Repräsentationsregime (2.2.1) und Prekarisierungsregime (2.2.2).

1 Im Türkischen kann «herkesin meydani» «Platz aller» bedeuten. 


\subsubsection{Verhältnis von Mahnmal und Repräsentationsregime}

Das Mahnmal macht Erfahrungen von Migrantinnen und Migranten nach den Anschlägen sichtbar, wie etwa die "sekundäre Viktimisierung» (Geschke und Quent 2016) durch Polizei, Behörden und Medien. Migrantische Opfer wurden als Täterinnen und Täter behandelt und so zum zweiten Mal zum Opfer gemacht (sinnbildlich hierfür ist das Wort «Dönermorde»). People of Color erschienen innerhalb dieser rassifizierten polizeilichen Anrufung pauschal als Sicherheitsrisiko ( $G$ Gefährder»). ${ }^{2}$ Indem das Mahnmal diese Täter-Opfer-Umkehr durch die Sicherheitsbehörden sichtbar macht, wird es zum Gegenraum des hegemonialen Blick- und Urteilsregimes und der rassifizierten (polizeilichen) Anrufungsordnung. Die dominanzgesellschaftliche Überformung der Prozesse der Enkodierung und Dekodierung von Symbolen, wie sie durch die polizeilichen Anrufungspraktiken vollzogen wurden, wird durch augmentierte migrantische Perspektiven mit Hilfe dieser materiell-diskursiven Gegenpraktiken überlagert und blossgestellt. Diese Abweichungen vom hegemonialen Repräsentationsregime eröffnen gerade durch die postdigitale Struktur des Mahnmals, die im gelebten Stadtraum die alltägliche Wahrnehmung stört, kontrahegemoniale Perspektiven. Als hybrider Akteur macht es die diskursiven Optimierungsprozesse deutlich, mit denen eine bessere Passung von bedeutungserzeugenden Praktiken an hegemoniale Massstäbe hergestellt wird und widersteht insofern den optimierenden Anpassungen der symbolischen Codierungsvollzüge durch das Repräsentationsregime.

Die politische Dimension medialer Apparate, so Stuart Hall, besteht in deren «Signifikationspolitik» (1982, 83). Informations- und Nachrichtenmedien sichern die Zirkulation hegemonialer Bedeutungen und gestatteten es, z. B. den Nagelbombenanschlag mit rassistischen Deutungen zu unterlegen («Dönermorde»). Das digitale Mahnmal kann insofern als gegenhegemonialer Signifikationsapparat verstanden werden. Die postdigitale Simulation des Hauses, also das Spiel mit augmentierten und nicht-augmentierten Räumen, ermöglicht Migrantinnen und Migranten, ihre eigene Sicht auf die Dinge darzustellen. Neben der Frage, was damals geschah und welche Folgen die Anschläge für die Opfer hatten, fragt das Mahnmal: Was wäre, wenn die Anschläge nicht stattgefunden hätten? Was wäre, wenn migrantisiertes Leben genau so viel Wert wäre wie deutsches? Was wäre, wenn die Mordserie gleich mit den richtigen Täterinnen und Tätern in Verbindung gebracht worden wäre? Wie kann eine Gesellschaft der Vielen aussehen?

Als gegenhegemonialer Signifikationsapparat fordert das digitale Mahnmal also dazu auf, sich in die Situation der Opfer zu versetzen und die migrantisierte Erfahrungsperspektive nachzuvollziehen. Die Keupstraße wird durch die im digital augmentierten Raum materialisierte Perspektivenübernahme decodiert und encodiert:

2 Eine solche Umkehr wurde zuletzt auch von Friedrich Merz (damals als Kanzlerkandidat der CDU) vertreten. Als Reaktion auf den rechtsterroristischen Anschlag von Hanau am 19. Februar 2020 forderte Merz eine stärkere Überwachung von Clan- und Ausländerkriminalität sowie schärfere Grenzkontrollen. 
die Signifikanten «Dönermorde», «türkische Mafia», «kriminelle Clans», «islamistische Gefährder» werden als Elemente hegemonialer Bedeutungsproduktion innerhalb der medialen Signifikationsapparate sichtbar gemacht. Medien, so kann mit Hall festgestellt werden, kommt insofern eine zentrale Rolle im «Kampf um Bedeutung» zu. Signifikationspolitik ist der Kampf um die Macht, ein bestimmtes Ereignis mit Bedeutung auszustatten und Medien sind auch noch in Prekarisierungsgesellschaften die dominanten Mittel sozialer Signifikation. Indem das digitale Mahnmal als materieller Signifikationsapparat eine antirassistische Bedeutungserzeugung sichert, entsubjektiviert es hegemoniale Selbstverhältnisse.

\subsubsection{Verhältnis von Mahnmal und Prekarisierungsregime}

Indem das Mahnmal auffordert, sich in die Situation der Opfer zu versetzen, appelliert es aber auch an uns, sich mit den Opfern zu solidarisieren. Mit der postkolonialen Theoretikerin María do Mar Castro Varela (2018) kann das Mahnmal als Raum kontrapunktischer Solidarität gedeutet werden. Der Kontrapunkt als musikalische Kompositionstechnik ermöglicht das Komponieren mehrstimmiger Gefüge, in denen jede Stimme ihre Eigenständigkeit wahrt und trotzdem ein Zusammenklang erzeugt wird. Trotz der Differenz der Stimmen wird es möglich, das Verschiedene musikalisch als Einheit zu hören. Die kompositionstechnische Metapher des Kontrapunkts dient Castro Varela als Bild für ein konflikthaftes Gemeinsam-Werden, in dem Differenzen nicht untergraben werden, sondern die Voraussetzung darstellen für eine, im Fall des Mahnmals, postdigitale solidarische Praxis. Wie in der kontrapunktischen Musizierpraxis entsteht auch im augmentierten Raum des Mahnmals eine Form solidarischer Gemeinsamkeit. Jede ${ }^{\star} r$ ist aufgefordert, zu sprechen und einzustimmen in den anhebenden kontrahegemonialen Diskurs. Es entsteht so ein Raum der Erzählungen, die sich dem bestehenden Narrativ der Opfer-Täter Umkehr durch die Sicherheitsbehörden widersetzt und den Status migrantisierten Lebens nicht auf den des Opfers reduziert. Indem so die Vielstimmigkeit des migrantisierten Lebens sichtbar wird, wird das Schweigen, das zuvor stattgefunden hat, durchbrochen.

Das Mahnmal als Raum kontrapunktischer Solidarität produziert Abweichungen gegenüber ausgewählten Direktiven des Prekarisierungsregimes. Zum einen fordert es im Sinne einer signifikationspolitischen Entsubjektivierung hegemonialer Selbstverhältnisse dazu auf, die Verknüpfung von Rassismus und individueller Absicherung zu erkennen und das selbstzentrierte Sicherheitsdispositiv als Verlust von Differenzgerechtigkeit zu begreifen. Zum anderen lädt es dazu ein, «neue Formen des Schutzes zu erfinden, die nicht in der immunisierenden Abwehr und Negation von Gefährdetheit und Kontingenz bestehen» (Lorey 2012, 140). Mit anderen Worten: Es fordert dazu auf, sich nicht mehr zur Sicherung mancher gegen bedrohliche Andere aufteilen und verteilen zu lassen und die eigene gefühlte Unsicherheit zu hinterfragen. Es geht darum zu erkennen, dass das bürgerliche Sicherheitsdispositiv darauf beruht, andere 
zum Sicherheitsrisiko zu erklären. Interventionen wie das Mahnmal sind insofern Kämpfe um Lebensverhältnisse und Denkweisen, mit denen das gouvernementale Spiel von Absicherung und Prekarisierung unterbrochen wird. Die kontrahegemoniale diskursive Praxis des Mahnmals sagt gewissermassen: Der radikale Individualismus und der Zwang, sich und das eigene Leben auf einem immer wieder gesenkten Mindestmass an Absicherung selbst zu gestalten und sich regierbar zu machen, muss nicht der alles bestimmende Massstab des Lebens sein. Das Mahnmal als hybrider Akteur konfrontiert Passantinnen und Passanten, die die Strasse durchqueren, mit den migrantischen Erfahrungen von Rechtsterrorismus und institutionellem Rassismus. Es fordert sie dazu auf, Teil der Vielen zu werden und kontrapunktisch an Gegenentwürfen mitzuwirken. Es eröffnet so die Vision eines Schutzes, der nicht auf einem individuell abgesicherten Leben aufbaut, wie es für das Optimierungsverständnis des Prekarisierungsregimes gilt. Das Mahnmal verkörpert diese andere Denkweise und widersteht in doppelter Hinsicht der servilen selbstoptimierenden «Kapitalisierung von Subjektivierungsweisen» (ebd.): einerseits als Raum der Sorge, im Sinne eines Sich-umeinander-Kümmerns und für andere Einstehens und andererseits als Raum der Vielen, d. h. als Praxis des Widerstehens gegen rassistische Differenzierungen und als prekäre Praxis kontrapunktischer Solidarität. Die Digitalisierung, die die Tendenz hat, sich mit dem Prekarisierungsregime zu verbinden und die Prozesse des Gegeneinander-Anordnens zu verstärken, wird hier zum Mittel andere Gesellschaftsentwürfe hervorzubringen. Das Mahnmal erinnert mittels seiner augmentierten Struktur nicht nur daran, welche Folgen an die individualisierende Sozialität der Optimierung geknüpft sind. Sie bringt auch temporäre Gegenräume hervor. Das sind Räume

a. gegen die Optimierung der individuellen Absicherung (Regime der Prekarisierung) und

b. gegen die Optimierung hegemonialer Repräsentationspraktiken, in denen migrantisierte Andere zur Bedrohung erklärt werden (Repräsentationsregime).

Es hält diesen Ordnungen ein radikaldemokratisches Ideal entgegen, das auf die «Lebbarkeit des Lebens» (Butler 2012), und zwar nicht nur des eigenen Lebens, zielt, sondern das sich gegen Zustände unlebbarer Prekarität im Allgemeinen richtet.

\section{Zusammenfassender Vergleich: Postdigitale Subjektivierungspraktiken und ihr Verhältnis zu Optimierung}

Aus den bisherigen Überlegungen lassen sich vier Vergleichskategorien extrahieren, die das herausgearbeitete Verhältnis der analysierten Beispiele zu den Optimierungsverständnissen des Repräsentationsregimes und des Regimes der Prekarisierung zu bestimmen erlauben (Tabelle 1). Wie sich anhand dieser vier Kategorien erläutern lässt, so lautet die Antwort auf unsere erste Forschungsfrage, spielen postdigitale 
Apparaturen eine ambivalente Rolle in Hinblick auf die Reproduktion von Macht- und Herrschaftsstrukturen. Wir hatten herausgearbeitet, dass das jeweils spezifische Subjektivierungsgeschehen in den Praxisbeispielen mit affirmatorischen bzw. widerständigen Praktiken im Kontext unterschiedlicher Formen der Optimierung verknüpft ist (A). In den Beispielen hatten wir nachgezeichnet, auf welche Weisen sich Subjekte in Vollzügen stets von neuem relational konstituieren und gleichzeitig durch postdigitale Praktiken, in die sie involviert sind, hervorgebracht werden. Dass es sich dabei um postdigitale Praktiken handelt, hatten wir erstens mit dem Verweis auf die vollzogene Unterminierung von Digitalität/Nichtdigitalität (B1) und zweitens anhand der kritischen Positionierung gegenüber zentralen Narrativen der Digitalisierung expliziert (B2). Die (in die postdigitale Praxis eingefaltete) Kritik trifft allerdings nur auf das Mahnmal als politische Praxis und nicht auf das Beispiel ‘Azuma) zu. Schliesslich hatten wir Postdigitalität als materiell-diskursive hybride Praxis bestimmt (C), die zu unterschiedlichen Regimen in Beziehung steht:

\section{A. Subjektivierung (im Verhältnis zu Regimen)}

‘Azuma Hikari: Im Beispiel zeigt sich ein serviles wie auch individualisiertes Subjektverhältnis. Zum einen haben wir es bei Azuma mit einem ‘subjektähnlichen) Assistenzsystem zu tun, das seine eigene Individualität und Handlungsspielräume innerhalb der Koordinaten eines vergeschlechtlichenden Repräsentationsregimes erhält, in dem es u.a. durch Sorgearbeiten zur ‘Anderen〉 der Selbstführungspraktiken des männlichen Erwerbssubjekts wird. Zum anderen nutzt der User die dadurch erzeugten Sicherheits- und Flexibilisierungsgewinne, um innerhalb des Regimes der Prekarisierung mit den wechselnden Anforderungen an die Optimierung des eigenen Selbstverhältnisses besser mithalten zu können.

Digitales Mahnmal: Subjektivierungsprozesse zeichnen sich sowohl im Rahmen des Repräsentationsregimes als auch im Regime der Prekarisierung durch ihren politisierenden Charakter aus. Im Repräsentationsregime konstituiert sich widerständige Subjektivität in der Abweichung von den beherrschenden bedeutungserzeugenden Diskursen als oppositionelle Bewegung. Im Verhältnis zum Regime der Prekarisierung entstehen politisierte Subjekte durch Assoziierung, indem sie sich der (z. B. rassialisierenden) Vereinzelung durch Formen der Kollektivierung widersetzen. Beide widerständige Bewegungen reartikulieren repressive Praktiken in kontrahegemonialer Absicht.

\section{B1. Postdigitalität (Verschränkung von Digitalität und Lebenswelt)}

‘Azuma Hikari): In Hinblick auf das in Vollzügen figurierte Subjekt-Technologie-Verhältnis überkreuzen sich die Positionen: Während das Assistenzsystem lernt, sich wie eine vergeschlechtlichte Andere zu verhalten und körperlich-emotionale Bedürfnisse des Anderen befriedigt sowie alltagsrelevante planerische Herausforderungen 
antizipiert, fördert die Einpassung in neoliberale Geschlechterlogiken den selbstkompetenten Umgang mit den gesellschaftlichen Anforderungen an flexible Erwerbssubjekte. Azuma und User synchronisieren auf diese Weise die Feedbackroutinen des bzw. der jeweils anderen. Auf der Ebene verkörperter Relationalität kommt es dabei zu einer Symmetrisierung. Beide brauchen den bzw. die jeweils andere(n) unter den Bedingungen der jeweiligen Praxisrelationen zu den Regimen des Prekären und der Repräsentation. Dieses symbiotische Verhältnis zwischen beiden simuliert wechselseitige Abhängigkeit voneinander und Ko-Exponiertheit innerhalb einer geteilten Lebenswelt. Auf diese Weise wird Gleichheit in einem hybriden Beziehungsgeflecht hergestellt, das aus einer vergeschlechtlichten Ungleichheitsordnung hervorgegangen ist.

Digitales Mahnmal: Hier zeigt sich die postdigitale Dimension des Technologieeinsatzes in der gezielten Infragestellung von bestehenden Herrschafts- und Gesellschaftsstrukturen. Dabei geht es a) darum, eine radikaldemokratische Praxis der Kontestation (Arendt 1986; Celikates 2010) zu ermöglichen, d. h. ausgeschlossene Widersprüche und Sichtweisen in den politisch-medialen Raum einzutragen. Und b) geht es um die politische Verknüpfung von Vereinzelten, die in einem asymmetrischen Regime rassialisierter Über- und Unterordnungsverhältnisse ständig bedroht werden. Zumeist ist diese Assoziation unmittelbar mit der Praxis der Kontestation verknüpft, insofern diese durch die Unterscheidung die Vereinzelten in ein Verhältnis zu den hegemonialen Verhältnissen setzt und damit neue Visionen von Gemeinschaft stiftet.

\section{B2. Postdigitalität (Kritik als Teil der Praxisvollzüge)}

‘Azuma Hikari): In den Relationen zwischen Azuma und User sind keine expliziten Möglichkeiten vorhanden, Kritik an dominanten Vorstellung als Bestandteil vergeschlechtlichender Praxisvollzüge zu äussern.

Digitales Mahnmal: Postdigitale Kontestationspraktiken und die solidarische Assoziation von Vereinzelten bieten Alternativen zur Optimierung von abwertenden Vorstellungen über Andere und zur prekarisierten Selbstoptimierung. Zu diesen Alternativen gehören die Vorstellung von einer konfliktorischen Repräsentationsordnung.

C. Verhältnis zwischen Regime und hybrider Praxis

(Azuma Hikari): Auf Basis des gendered-deep-learning werden ausgewählte Sorgearbeiten verrichtet und Weiblichkeit in einer Weise verkörpert, die sowohl zu männlichen Erwartungen als auch zur materiellen Dimension von vergeschlechtlichter Arbeitsteilung in prekären Regimen passt. Gleichzeitig wird über die Aspekte der Synchronisierungs- und Symmetrisierungspraxis ein männliches Subjekt der Selbstführung hervorgebracht, das die eigene Abhängigkeit von sorgenden Anderen 
anerkennt. Eigene und von Azuma geäusserte emotionale Bedürfnisse lernt der User zu artikulieren bzw. zu erkennen. Im Gegensatz zur hegemonial-männlichen Verkörperung von Subjektivität, die sich betont unabhängig von Anderen und von der eigenen Vulnerabilität gibt (vgl. Connell 2015), wird ein fragiles Erwerbssubjekt verkörpert, das die Bedingungen der Sorge um die eigene Prekarität anderen zuweist.

Digitales Mahnmal: Hier geht es um die Realisierung zweier politischer Ziele: es geht zum einen darum, die Schuld für die eigenen Lebensbedingungen nicht allein bei sich zu suchen, sondern ihren Zusammenhang zur Prekarisierung der Lebensverhältnisse zu erkennen. Prekarisierungsdynamiken können zeitweise unterbrochen werden, indem man sich nicht zur Sicherung mancher gegen bedrohliche Andere aufteilen und verteilen lässt. Andererseits geht es darum, die beherrschende rassistische Signifikationspolitik zu durchkreuzen. Medien als die dominanten Mittel sozialer Signifikation sichern Bedeutungserzeugung, indem z. B. die Anschläge des NSU mit dem Signifikant «Dönermorde» in Verbindung gebracht wurden. Das digitale Mahnmal als materieller Signifikationsapparat ermöglicht eine antirassistische Bedeutungserzeugung und dadurch die hegemonialen rassistischen Selbstverhältnisse zu entsubjektivieren.

\begin{tabular}{|l|l|l|}
\hline Analysebeispiel & 〈Azuma Hikari` & Digitales Mahnmal \\
\hline A. Subjektivierung & servil-individualisierend & oppositionell-kollektivierend \\
\hline B1. Postdigitalität 1 & Synchronisierung (symmetrisch) & Assoziierung (kontestativ) \\
\hline B2. Postdigitalität 2 & Nein & Ja \\
\hline $\begin{array}{l}\text { C. Verhältnisse zwi- } \\
\text { schen Regime und } \\
\text { hybrider Praxis }\end{array}$ & $\begin{array}{l}\text { Beziehungen zwischen gendered } \\
\text { deep learning und davon profi- } \\
\text { tierendem «fragilen` männlichen } \\
\text { Selbstunternehmer (‘Selbstcybor- } \\
\text { gisierung`) }\end{array}$ & $\begin{array}{l}\text { Entsubjektivierung hegemonialer } \\
\text { Selbstverhältnisse, sich nicht der- } \\
\text { massen aufteilen und verteilen zu } \\
\text { lassen }\end{array}$ \\
\hline
\end{tabular}

\section{Literatur}

Allert, Heidrun, Michael Asmussen, und Christoph Richter. 2018. «Formen von Subjektivierung und Unbestimmtheit im Umgang mit datengetriebenen Lerntechnologien - eine praxistheoretische Position». Zeitschrift für Erziehungswissenschaft 21 (1): 142-58. https://doi. org/10.1007/s11618-017-0778-7.

Arendt, Hannah. 1986. "Ziviler Ungehorsam». In Zur Zeit. Hannah Arendt. Politische Essays (1943-1975), herausgegeben von Marie Luise Knott, 119-161. Berlin: Rotbuch. 
Bettinger, Patrick. 2020. «Materialität und digitale Medialität in der erziehungswissenschaftlichen Medienforschung. Ein praxeologisch-diskursanalytisch perspektivierter Vermittlungsversuch». In Jahrbuch Medienpädagogik 15: Erziehungswissenschaftliche und medienpädagogische Online-Forschung: Herausforderungen und Perspektiven, herausgegeben von Johannes Fromme, Stefan Iske, Therese Leik, Steffi Rehfeld, Jasmin Bastian, Manuela Pietraß, und Klaus Rummler, 15-34. Zürich: Zeitschrift MedienPädagogik, Sektion Medienpädagogik (DGfE). https://doi.org/10.21240/mpaed/jb15/2020.03.04.X.

Butler, Judith. 2012. «Kann man ein gutes Leben im schlechten führen? Judith Butlers Dankesrede». Frankfurter Rundschau, 15. September 2012, Abschn. Kultur. https://www.fr.de/ kultur/kann-gutes-leben-schlechten-fuehren-11319646.html.

Castro Varela, M. 2018. «Bildet euch, denn wir brauchen all eure Klugheit» Erwachsenenbildung und kontrapunktische Solidarität». In Dokumentation der Tagung „Gegen den Strich. Solidarität in der Erwachsenenbildung, 29. - 30. Mai 2018, St. Wolfgang, Österreich, 18-30, https://www.bifeb.at/fileadmin/user_upload/doc/gegen_den_strich_dokumentation.pdf.

Celikates, Robin. 2010. «Ziviler Ungehorsam und radikale Demokratie. Konstitutive vs. konstituierte Macht». In Das Politische und die Politik, herausgegeben von Thomas Bedorf and Kurt Röttgers, 274-302. Frankfurt a. M.: Suhrkamp.

Connell, Raewyn. 2015. Der gemachte Mann. Konstruktion und Krise von Männlichkeiten. Wiesbaden: Springer VS. https://doi.org/10.1007/978-3-531-19973-3.

Crawford, Kate. 2016. «Artificial Intelligence's White Guy Problem». The New York Times, 25. Juni 2016, Abschn. Opinion. https://www.nytimes.com/2016/06/26/opinion/sunday/artificial-intelligences-white-guy-problem.html.

Dander, Valentin. 2020. «Sechs Thesen zum Verhältnis von Bildung, Digitalisierung und Digitalisierung». In Digitalisierung - Subjekt - Bildung: Kritische Betrachtungen der digitalen Transformation, herausgegeben von Valentin Dander, Patrick Bettinger, Estella Ferraro, Christian Leineweber, und Klaus Rummler, 19-37. Opladen: Verlag Barbara Budrich. https://doi.org/10.2307/j.ctvvb7n3h.

Dimitrova, Petja, Eva Egermann, Tom Holert, Jens Kastner, und Johanna Schaffner. 2012. Regime. Wie Dominanz organisiert und Ausdruck formalisiert wird. Münster: Ed. Assemblage.

Eubanks, Virginia. 2018. Automating Inequality. How High-Tech Tools Profile, Police, and Punish the Poor. Maidenhead: Melia Publishing.

Geschke, Daniel, und Matthias Quent. 2016. «Sekundäre Viktimisierung durch die Polizei?» In Rechtsextremismus und „Nationalsozialistischer Untergrund“: Interdisziplinäre Debatten, Befunde und Bilanzen, herausgegeben von Wolfgang Frindte, Daniel Geschke, Nicole Haußecker, und Franziska Schmidtke, 481-505. Edition Rechtsextremismus. Wiesbaden: Springer Fachmedien. https://doi.org/10.1007/978-3-658-09997-8_21.

Hall, Stuart. 1982. "The Rediscovery of 'Ideology': Return of the Repressed in Media Studies». In Culture, Society and the Media, herausgegeben von Tony Bennett, James Curran, Michael Gurevitch, und Janet Wollacott, 56-90. London: Routledge. https://doi. org/10.4324/9780203978092. 
Hall, Stuart. 1994. Die Frage der kulturellen Identität. In Rassismus und kulturelle Identität. Ausgewählte Schriften 2, edited by Ulrich Mehlem, Dorothee Bohle, Joachim Gutsche, Matthias Oberg and Dominik Schrage, 180-222. Hamburg: Argument.

Hark, Sabine, und Paula-Irene Villa. 2017. Unterscheiden und herrschen: Ein Essay zu den ambivalenten Verflechtungen von Rassismus, Sexismus und Feminismus in der Gegenwart. Bielefeld: transcript Verlag. https://doi.org/10.14361/9783839436530.

Knox, Jeremy. 2019. «What Does the 'Postdigital' Mean for Education? Three Critical Perspectives on the Digital, with Implications for Educational Research and Practice». Postdigital Science and Education 1 (2): 357-70. https://doi.org/10.1007/s42438-019-00045-y.

Lorey, Isabell. 2012. Die Regierung der Prekären. Wien, Berlin.

Marchart, Oliver. 2008. Cultural Studies. Konstanz: UVK UTB.

Müller, Oliver. 2010. Zwischen Mensch und Maschine. Vom Glück und Unglück des Homo Faber. Berlin: Suhrkamp.

Penny, Laurie. 2016. «Why do we give robots female names? Because we don't want to consider their feelings». NewStatesman, 22 April 2016. https://www.newstatesman.com/politics/feminism/2016/04/why-do-we-give-robots-female-names-because-we-dont-wantconsider-their.

Ranga, Marina, und Henry Etzkowitz. 2010. «Athena in the World of Techne: The Gender Dimension of Technology, Innovation and Entrepreneurship». Journal of Technology Management \& Innovation 5 (1): 1-12. https://doi.org/10.4067/S0718-27242010000100001.

Ronald, Richard, und Yosuke Hirayama. 2009. «Home Alone: The Individualization of Young, Urban Japanese Singles». Environment and Planning A: Economy and Space 41 (12): 283654. https://doi.org/10.1068/a41119.

Sternfeld, Nora. 2020. «Eine Demokratie lernen, die es noch nicht gibt». In Making Democracy - Aushandlungen von Freiheit, Gleichheit und Solidarität im Alltag, herausgegeben von Elke Rajal, Oliver Marchart, Nora Landkammer, und Carina Maier, 205-14. Bielefeld: transcript Verlag. https://doi.org/10.14361/9783839450161-017.

Said, Edward. 1978. Orientalism. New York: Pantheon Books.

Terranova, Tiziana. 2000. «Free Labor: Producing Culture for the Digital Economy». Social Text 18 (2): 33-58. https://muse.jhu.edu/article/31873.

Vasconcelos, Elvia. 2018. «Gendering Personal Intelligent Assistants». Elvia's Blog of Everything (blog). 2. Mai 2018. https://elviavasconcelosblog.wordpress.com/2018/05/02/genderingpersonal-intelligent-assistants/.

Weber, Jutta, und Corinna Bath. 2007. «'social' Robots \& 'Emotional' Software Agents: Gendering Processes and De-Gendering Strategies for 'Technologies in the Making'». In Gender Designs IT: Construction and Deconstruction of Information Society Technology, herausgegeben von Isabel Zorn, Susanne Maass, Els Rommes, Carola Schirmer, und Heidi Schelhowe, 53-63. Wiesbaden: VS Verlag für Sozialwissenschaften. https://doi.org/10.1007/978-3-53190295-1_3. 